\title{
Development and characterization of genomic simple sequence repeats for Colocasia gigantea (Blume) Schott using 454 sequencing
}

\author{
Yujing Liư ${ }^{1,3}$, Yu Guo², Deke Xing ${ }^{1}$, and Chunlin Long ${ }^{*}$ \\ ${ }^{1}$ Jiangsu University, College of Agricultural Equipment and Engineering, Zhenjiang 212013, PR China. \\ ${ }^{2}$ BGI Genomics, BGI-Shenzhen, Shenzhen 518083, China. \\ ${ }^{3}$ Minzu University of China, College of Life and Environmental Sciences, Beijing 100081, China. \\ *Corresponding author (long@mail.kib.ac.cn).
}

Received: 25 July 2017; Accepted: 29 October 2017; doi:10.4067/S0718-58392018000100023

\begin{abstract}
The petiole of Colocasia gigantea (Blume) Schott is an important agricultural and biological organ, which contains high dietary fiber, pyridoxine, and nicotinamide. However, available genomic resources of C. gigantea are scarce, and this restricts further genetic diversity research, linkage map construction, and marker-assisted selection in $C$. gigantea. A large-scale genomic DNA study of $C$. gigantea was conducted using the 454 sequencing technology to develop simple sequence repeats (SSRs). We identified 31069 putative genomic $C$. gigantea SSRs, and 100 primers were randomly selected to validate their usefulness in $10 \mathrm{C}$. gigantea samples. The specificity of six primers yielded amplification products with expected sizes and exhibited polymorphism. The number of alleles per locus ranged from 3 to 7 alleles and the polymorphic information content (PIC) ranged from 0.561 to 0.756 . The newly developed SSRs in this study should be useful tools for assessing genetic diversity, understanding population structure, and conserving and using C. gigantea effectively.
\end{abstract}

Key words: Microsatellite markers, 454 sequencing.

\section{INTRODUCTION}

Colocasia gigantea (Blume) Schott, a member of the monocotyledonous family Araceae, is an important food crop in Southeast Asia. It is widely planted throughout most wet tropical and subtropical regions, including Vietnam, China, and Japan, for sustainable subsistence. Colocasia gigantea is cultivated for its petiole, which is consumed as a vegetable (Nguyen, 2005; Ivancic et al., 2008). Sometimes individual leaves are used to make soup, and the corms and leaves are also used for medicinal purposes. Colocasia gigantea is now commercially cultivated in many minority regions of China and the petiole is sold in local markets. In Hawaii, besides local consumption, C. gigantea is exported to the rest of the United States, France, and Japan to meet the cultural nutritional needs of Vietnamese migration (Nguyen, 2005). Aroids are commonly acrid (Bown, 2000) and require plant material to be processed before it can be edible; they are usually cooked to remove acridity. Wild $C$. gigantea cannot be eaten; however, cultivated $C$. gigantea can be eaten, which suggests its high genetic diversity and long history of human selection and cultivation. Although China has a long history of consuming $C$. gigantea, the $C$. gigantea genetic resource is still scarce. Genetic studies will offer great potential to detect allelic forms of a gene and phenotypes and accelerate the progress of $C$. gigantea research.

Simple sequence repeats (SSRs) are one of the most informative and versatile DNA-based markers used in plant genetic research because of their high polymorphism, abundance, co-dominance, and short length, they include 
linkage map development, quantitative trait locus (QTL) mapping, marker-assisted selection, parentage analysis, cultivar fingerprinting, genetic diversity, gene flow, and evolutionary studies (Zalapa et al., 2012). For plants without a genome sequence, developing SSR primers is a very challenging job. The traditional method of isolating microsatellites is time-consuming and cost-intensive and has a relatively low efficiency in microsatellite detection. Many studies have indicated that next-generation sequencing (NGS) is an efficient method to identify large numbers of microsatellites at a fraction of the cost and effort of traditional approaches (Angeloni et al., 2011; Ho et al., 2011; Baldwin et al., 2012; Georgi et al., 2012; Kale et al., 2012; Perry and Rowe, 2011; Sahu et al., 2012; Yang et al., 2012). The two major NGS platforms with an emergent application of SSRs are 454 and Illumina. Compared to Illumina, the advantages of 454 are longer read lengths, higher consensus accuracy, and faster run times (Egan et al., 2012; Zalapa et al., 2012); Illumina is mainly limited to organisms with available reference genomes (Wang et al., 2010). In the present study, we chose NGS (Roche 454 GS FLX+) to develop SSRs and search for highpolymorphism SSRs that can be used for genetic diversity.

This is the first study developing $C$. gigantea SSRs; it will help to evaluate genetic diversity, linkage map construction, marker-assisted selection, and other related studies of $C$. gigantea and species of Araceae.

\section{MATERIALS AND METHODS}

\section{Plant materials and DNA isolation}

The leaf samples were collected from 10 individual healthy $C$. gigantea plants (four wild and six cultivated). Detailed information of all plant materials used in the present study is listed in Table 1. For 454 sequencing, total genomic DNA was isolated from the petiole tissues using the $4 \times$ CTAB protocol (Dellaporta et al., 1983). The DNA products were then visualized by electrophoresis on SYBR Green I stained 2.0\% agarose gel with Trans DNA marker I (Ding Guo Co., Beijing, China) to evaluate quality. DNA samples were stored at $-20{ }^{\circ} \mathrm{C}$, and DNA integrity was verified with a Bioanalyzer (2100, Agilent, Santa Clara, California, USA) prior to sequencing. Approximately $5 \mu \mathrm{g}$ genomic DNA was sequenced with a GS FLX ${ }^{+}$platform (Roche Applied Science, Indianapolis, Indiana, USA). Sequencing was carried out according to the manufacturer's protocol in BGI Genomics (Shenzhen, China).

\section{Polymerase chain reaction (PCR) conditions}

Polymerase chain reaction (PCR) amplifications were carried out with a MyCycler Thermal Cycler (Bio-RAD, Hercules, California, USA) in a final volume of $20 \mu \mathrm{L}$. Each reaction tube contained $2 \mu \mathrm{L}$ PCR buffer, $2 \mu \mathrm{L}$ dNTP, $2 \mu \mathrm{L}$ of each primer, $1 \mu \mathrm{L}$ genomic DNA, $2 \mu \mathrm{L}$ Taq DNA polymerase (Fermentas, Vilnius, Lithuania), and $10 \mu \mathrm{L}$ distilled water. For the PCR reaction program, amplification conditions in the thermocycler were as follows: preincubation at $94{ }^{\circ} \mathrm{C}$ for 5 min followed by 35 cycles of denaturation at $94{ }^{\circ} \mathrm{C}$ for $40 \mathrm{~s}$, annealing at $55^{\circ} \mathrm{C}$ (depending on primer pairs) for $45 \mathrm{~s}$, elongation at $72{ }^{\circ} \mathrm{C}$ for $30 \mathrm{~s}$, and a final extension step at $72{ }^{\circ} \mathrm{C}$ for 5 min. Optimized SSR primers were used to amplify DNA from five plant individuals to select polymorphic microsatellite markers.

Table 1. Origin of Colocasia gigantea plant materials used in the present study.

\begin{tabular}{rrrrl}
\hline $\mathrm{Nr}$ & Latin name & Characteristics & Part of collection & \multicolumn{1}{c}{ Locality } \\
\hline 1 & C.gigantea & Wild & Leaf & China, Xishuangbanna \\
2 & C.gigantea & Wild & Leaf & China, Hunan \\
3 & C.gigantea & Wild & Leaf & China, Guangxi \\
4 & C.gigantea & Wild & Leaf & China, Kunming \\
5 & C.gigantea & Cultivar & Leaf & China, Xishuangbanna \\
6 & C.gigantea & Cultivar & Leaf & China, Xishuangbanna \\
7 & C.gigantea & Cultivar & Leaf & China, Hunan \\
8 & C.gigantea & Cultivar & Leaf & China, Hunan \\
9 & C.gigantea & Cultivar & Leaf & China, Guangxi \\
10 & C.gigantea & Cultivar & Leaf & China, Kunming \\
\hline
\end{tabular}




\section{Sequence analysis and identification of simple sequence repeats (SSRs)}

Before assembly, we carried out a stringent filtering process of raw sequencing reads. Only the sequences containing mitochondrial- or plastid-encoded genes were discarded. We removed 454 adaptor sequences from the raw reads obtained with the GS-FLX Titanium sequencer. Only high-quality reads that were at least 50 nucleotides in length were included in further analyses. In the present study, Newbler v2.5 (Roche/454 Life Sciences, Branford, Connecticut, USA) was used to cluster and assemble high-quality reads into a set of non-redundant contigs. Thresholds chosen for the aggregation steps were identified at $90 \%$ with a minimum overlapping length of 40 nucleotides (Li et al., 2012). We only searched for SSRs from nuclear genome using the MISA software (http://pgrc.ipk-gatersleben.de/misa/). The minimum length for each type of SSR was set as mononucleotide repeats $\geq 10$ nucleotides, di-nucleotide repeats $\geq 12$ nucleotides, tri-nucleotide repeats $\geq 15$ nucleotides; tetra-nucleotide repeats $\geq 16$ nucleotides, penta-nucleotide repeats $\geq 20$ nucleotides, and hexa-nucleotide repeats $\geq$ 24 nucleotides. Oligo-nucleotide primers were designed for selected SSR loci (where repeats were located at least $50 \mathrm{bp}$ from the 5' and 3' end of the sequences) using the PRIMER3 software (http://frodo.wi.mit.edu/primer3/). The parameters for primer design were preferred amplicon size of 100-200 bp, primer size 18-27 bp, and primer melting temperature of $55-60{ }^{\circ} \mathrm{C}$; the optimum temperature was $55^{\circ} \mathrm{C}$. When none of the three criteria could be met, the priority was the melting temperature. All primers were synthesized by Invitrogen (Invitrogen Co., Shanghai, China).

\section{Simple sequence repeat (SSR) marker validation and polymorphic SSR selection}

Genomic DNA from C. gigantea was extracted as described above and diluted prior to being used as templates to select polymorphic loci. Polymerase chain reactions were also performed as described above. Products from the amplification reactions were resolved on PAGE gels consisting of $4.5 \%$ polyacrylamide and $7 \mathrm{M}$ urea in $0.5 \times \mathrm{TBE}$ buffer, and the fragments were subsequently visualized by silver staining. The statistical analyses of SSR data were performed with PowerMarker 3.25 software (http://www.powermarker.net). The allelic polymorphism information content (PIC) was calculated by the following formula: PIC $=1-\sum\left(P_{i}\right)^{2}$ where $\mathrm{P}_{\mathrm{i}}$ is the SSR $\mathrm{i}^{\text {th }}$ allele frequency (Botstein et al., 1980). Polymorphism statistics, including allele number, observed/expected heterozygosities, inbreeding coefficient, and linkage equilibrium between loci, were calculated with GENEPOP v4.2 (Raymond and Rousset, 1995).

\section{RESULTS}

\section{Roche GS FLX 454 sequencing and assembly}

We obtained 1232789 reads with a total length of $621787375 \mathrm{bp}$. We incorporated 1043587 reads (84.7\%) into 46234 contigs and 630430 singletons for a total of 676664 unique sequences. The rest of the trimmed raw reads (189 202; 15.3\%) were excluded from the assembly because the sequences were too short, chimeric, or contained repetitive segments. The contigs had a maximum length of 71068 nucleotides. Among all contigs, 100-200 bp was the most frequent.

\section{Simple sequence repeat (SSR) identification and characterization}

We identified 31069 putative microsatellites. The frequencies of short tandem repeats, categorized by their unit sizes and the number of repeats, are summarized in Figure 1. Mono-nucleotide repeats $(26$ 064, 83.9\%) comprise the largest group of repeat motifs followed by di-nucleotide repeats $(4904,15.8 \%)$, while tetra-nucleotide repeats and penta-nucleotide repeats were the lowest (Figure 1).

Analysis of $\mathrm{A} / \mathrm{T}$ and $\mathrm{C} / \mathrm{G}$ mono-nucleotide repeats showed that the $\mathrm{A} / \mathrm{T}$ motif was significantly overrepresented (71.17\%) in C. gigantea genomic sequences (Table 2 ). The $\mathrm{A} / \mathrm{T}$ repeats were not the only predominant mononucleotide repeats, but they were also the most frequent motif in the entire genome, representing $59.71 \%$ of total SSRs. Among the di-nucleotide tandem repeats, GA/TC and AG/CT were by far the most common and accounted for $31.749 \%$ and $31.505 \%$ of all di-nucleotide motifs, respectively; this was followed by AT/TA (18.94\%), whereas the GT/AC repeat was extremely rare (8.667\%; Table 2$)$. The majority of tri-nucleotide repeats found in genomic sequences were TTC, which represented $17.17 \%$ of all tri-nucleotide repeats, followed by TTG (11.11\%; Table 2). With respect to tetra-nucleotide repeats, we only identified ATAC in the $C$. gigantea genome (Table 2). 
Figure 1. Distribution of various classes of simple repeat motifs with different numbers of repeats in the Colocasia gigantea genome.

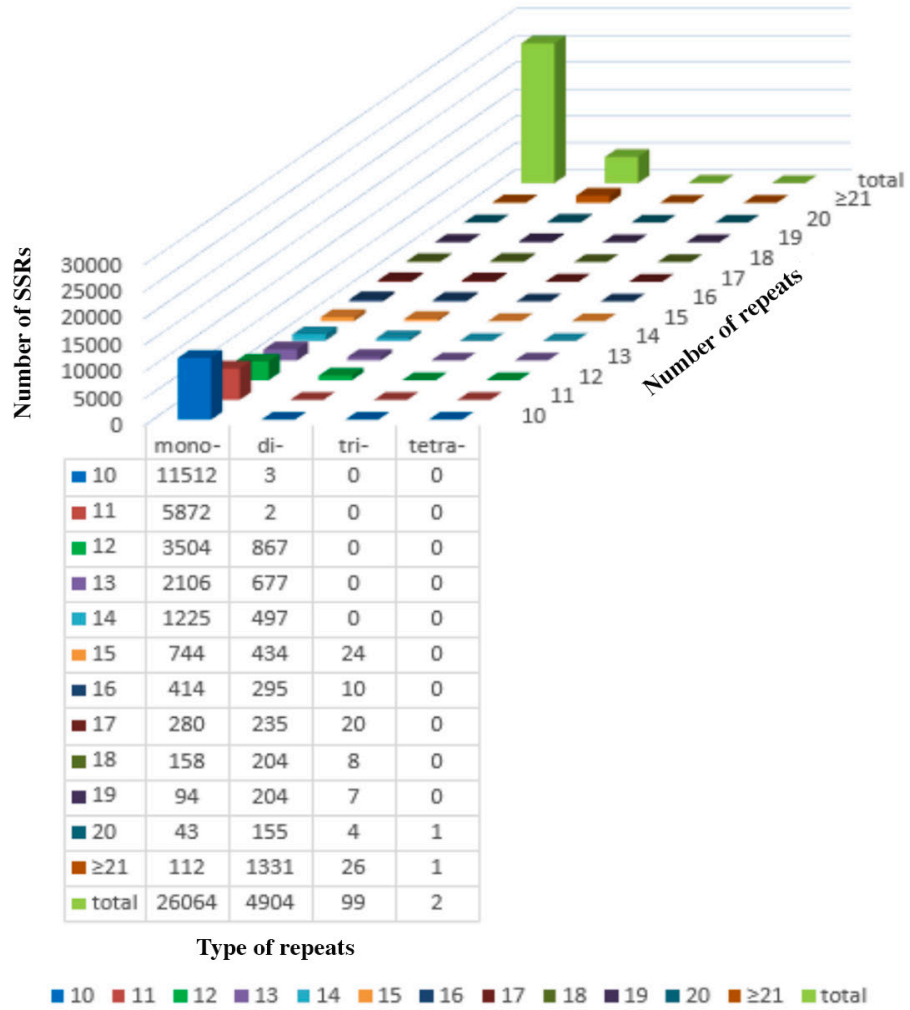

SSR: Simple sequence repeat.

\section{Marker validation and polymorphic simple sequence repeat (SSR) selection}

To assess the practicality of the microsatellites discovered in silico, 100 non-redundant genomic SSRs containing sequences (10 mono-, 70 di-, and 20 tri-nucleotide repeats) were randomly selected among 10 C. gigantea samples. Seventy-eight primers (89\%) were successfully amplified. Of these amplifiable loci, 36 yielded specificity amplification. Of these 36 loci, 12 yielded products of expected sizes and 24 primers yielded at least two bands. Of these 12 markers, 6 microsatellite markers were scorable and exhibited a broad range of allelic diversity among examined $C$. gigantea. The list of six sequences containing polymorphic SSRs was deposited in GenBank (KR010361-KR010366) (Table 3). For each of the SSR markers, both the forward and reverse primer sequences and PCR product sizes of $C$. gigantea are listed in Table 3. For the six polymorphic loci, the number of alleles per locus ranged from 3 to 7 alleles. We identified 28 alleles, with a mean of 2.8 alleles per locus. The PIC ranged from 0.561 to 0.756 (Table 4).

\section{Data access}

The raw sequence data for $C$. gigantea can be accessed at the National Center for Biotechnology Information (NCBI), Sequence Read Archive (SRA) (NCBI, Bethesda, Maryland, USA) with ID SRR6206491. The sample accession number is SAMN07786806. The BioProject number is PRJNA414351.

\section{DISCUSSION}

The identification and development of molecular markers represent significant challenges, especially for organisms that have little or no genomic sequence information. We used next-generation and high-throughput sequencing technology to develop additional genomic-derived microsatellite markers in $C$. gigantea

The genome sequences obtained in the present study were incomplete; therefore, the reported 31069 putative genomic-derived SSRs could be underestimated. We discovered that a large proportion of mono-nucleotide repeats 
Table 2. Frequencies of non-redundant simple sequence repeat motifs with respect to repeat numbers.

\begin{tabular}{|c|c|c|c|c|c|c|c|c|c|c|c|c|c|c|}
\hline Repeat Motif & 10 & 11 & 12 & 13 & 14 & 15 & 16 & 17 & 18 & 19 & 20 & $>20$ & Total & $\%$ \\
\hline A & 4508 & 2083 & 1030 & 601 & 317 & 192 & 75 & 66 & 28 & 16 & 6 & 19 & 8941 & 34.30 \\
\hline $\mathrm{C}$ & 924 & 786 & 624 & 368 & 159 & 57 & 9 & 7 & 2 & 1 & 0 & 11 & 2948 & 11.31 \\
\hline $\mathrm{T}$ & 5019 & 2097 & 1094 & 580 & 348 & 202 & 130 & 77 & 32 & 14 & 3 & 13 & 9609 & 36.87 \\
\hline G & 1061 & 906 & 756 & 557 & 401 & 293 & 200 & 130 & 96 & 63 & 34 & 69 & 4566 & 17.52 \\
\hline Monomeric (total) & 11512 & 5872 & 3504 & 2106 & 1225 & 744 & 414 & 280 & 158 & 94 & 43 & 112 & 26064 & 100.00 \\
\hline $\mathrm{AC}$ & 2 & 1 & 46 & 35 & 22 & 19 & 11 & 15 & 9 & 6 & 6 & 118 & 290 & 5.914 \\
\hline $\mathrm{AG}$ & 0 & 0 & 113 & 100 & 114 & 93 & 84 & 71 & 78 & 74 & 74 & 414 & 1215 & 24.776 \\
\hline AT & 0 & 0 & 186 & 151 & 93 & 81 & 33 & 29 & 9 & 2 & 2 & 1 & 587 & 11.970 \\
\hline $\mathrm{CA}$ & 1 & 1 & 38 & 30 & 11 & 17 & 12 & 11 & 3 & 6 & 5 & 124 & 259 & 5.281 \\
\hline $\mathrm{CT}$ & 0 & 0 & 109 & 71 & 45 & 29 & 25 & 13 & 6 & 8 & 3 & 21 & 330 & 6.729 \\
\hline GA & 0 & 0 & 99 & 94 & 91 & 92 & 72 & 65 & 79 & 87 & 60 & 518 & 1257 & 25.632 \\
\hline GT & 0 & 0 & 38 & 19 & 10 & 8 & 4 & 4 & 0 & 4 & 0 & 48 & 135 & 2.753 \\
\hline $\mathrm{TA}$ & 0 & 0 & 95 & 91 & 61 & 48 & 25 & 10 & 6 & 4 & 0 & 2 & 342 & 6.974 \\
\hline $\mathrm{TC}$ & 0 & 0 & 110 & 62 & 38 & 26 & 15 & 12 & 9 & 7 & 2 & 19 & 300 & 6.117 \\
\hline TG & 0 & 0 & 33 & 24 & 12 & 21 & 14 & 5 & 5 & 6 & 3 & 66 & 189 & 3.854 \\
\hline Dimeric (total) & 3 & 2 & 867 & 677 & 497 & 434 & 295 & 235 & 204 & 204 & 155 & 1331 & 4904 & 100.00 \\
\hline AAC & 0 & 0 & 0 & 0 & 0 & 0 & 0 & 1 & 1 & 0 & 0 & 0 & 2 & 2.02 \\
\hline AAT & 0 & 0 & 0 & 0 & 0 & 1 & 0 & 1 & 2 & 0 & 1 & 0 & 5 & 5.05 \\
\hline ACA & 0 & 0 & 0 & 0 & 0 & 0 & 1 & 2 & 0 & 1 & 0 & 3 & 7 & 7.07 \\
\hline $\mathrm{ACT}$ & 0 & 0 & 0 & 0 & 0 & 0 & 1 & 0 & 0 & 0 & 0 & 0 & 1 & 1.01 \\
\hline AGA & 0 & 0 & 0 & 0 & 0 & 0 & 0 & 0 & 0 & 1 & 0 & 0 & 1 & 1.01 \\
\hline AGT & 0 & 0 & 0 & 0 & 0 & 0 & 0 & 1 & 0 & 0 & 0 & 1 & 2 & 2.02 \\
\hline ATC & 0 & 0 & 0 & 0 & 0 & 0 & 0 & 0 & 0 & 0 & 0 & 1 & 1 & 1.01 \\
\hline ATG & 0 & 0 & 0 & 0 & 0 & 0 & 0 & 0 & 0 & 0 & 0 & 1 & 1 & 1.01 \\
\hline ATT & 0 & 0 & 0 & 0 & 0 & 0 & 0 & 1 & 0 & 0 & 0 & 0 & 1 & 1.01 \\
\hline CAA & 0 & 0 & 0 & 0 & 0 & 2 & 1 & 2 & 0 & 0 & 0 & 2 & 7 & 7.07 \\
\hline CAT & 0 & 0 & 0 & 0 & 0 & 1 & 2 & 0 & 0 & 0 & 1 & 0 & 4 & 4.04 \\
\hline CCA & 0 & 0 & 0 & 0 & 0 & 0 & 0 & 1 & 0 & 0 & 0 & 0 & 1 & 1.01 \\
\hline CTT & 0 & 0 & 0 & 0 & 0 & 3 & 0 & 1 & 0 & 0 & 1 & 0 & 5 & 5.05 \\
\hline GAT & 0 & 0 & 0 & 0 & 0 & 0 & 0 & 0 & 0 & 0 & 0 & 4 & 4 & 4.04 \\
\hline GGT & 0 & 0 & 0 & 0 & 0 & 0 & 0 & 0 & 0 & 0 & 0 & 1 & 1 & 1.01 \\
\hline GTA & 0 & 0 & 0 & 0 & 0 & 0 & 0 & 0 & 1 & 0 & 0 & 0 & 1 & 1.01 \\
\hline GTT & 0 & 0 & 0 & 0 & 0 & 1 & 0 & 0 & 0 & 0 & 0 & 0 & 1 & 1.01 \\
\hline TAA & 0 & 0 & 0 & 0 & 0 & 1 & 0 & 0 & 0 & 0 & 0 & 0 & 1 & 1.01 \\
\hline TAC & 0 & 0 & 0 & 0 & 0 & 0 & 0 & 2 & 0 & 0 & 0 & 0 & 2 & 2.02 \\
\hline TCA & 0 & 0 & 0 & 0 & 0 & 2 & 0 & 0 & 1 & 0 & 0 & 2 & 5 & 5.05 \\
\hline TCG & 0 & 0 & 0 & 0 & 0 & 0 & 1 & 0 & 0 & 0 & 0 & 0 & 1 & 1.01 \\
\hline TCT & 0 & 0 & 0 & 0 & 0 & 2 & 3 & 2 & 1 & 0 & 1 & 0 & 9 & 9.09 \\
\hline TGA & 0 & 0 & 0 & 0 & 0 & 1 & 1 & 0 & 0 & 0 & 0 & 3 & 5 & 5.05 \\
\hline TGG & 0 & 0 & 0 & 0 & 0 & 0 & 0 & 0 & 0 & 1 & 0 & 0 & 1 & 1.01 \\
\hline TGT & 0 & 0 & 0 & 0 & 0 & 0 & 0 & 1 & 0 & 0 & 0 & 0 & 1 & 1.01 \\
\hline TTA & 0 & 0 & 0 & 0 & 0 & 1 & 0 & 0 & 0 & 0 & 0 & 0 & 1 & 1.01 \\
\hline TTC & 0 & 0 & 0 & 0 & 0 & 6 & 0 & 1 & 1 & 3 & 0 & 6 & 17 & 17.17 \\
\hline TTG & 0 & 0 & 0 & 0 & 0 & 3 & 0 & 4 & 1 & 1 & 0 & 2 & 11 & 11.11 \\
\hline Trimeric (total) & 0 & 0 & 0 & 0 & 0 & 24 & 10 & 20 & 8 & 7 & 4 & 26 & 99 & 100.00 \\
\hline ATAC & 0 & 0 & 0 & 0 & 0 & 0 & 0 & 0 & 0 & 0 & 1 & 1 & 2 & 100.00 \\
\hline Tetrameric (total) & 0 & 0 & 0 & 0 & 0 & 0 & 0 & 0 & 0 & 0 & 1 & 1 & 2 & 100.00 \\
\hline
\end{tabular}

were AT-rich (Table 2). A similar occurrence was observed in Hevea brasiliensis (Li et al., 2012). The prevalence of genomic-derived SSRs with AT dimeric repeats has also been reported for H. brasiliensis (Li et al., 2012), Pinus massoniana (Bai et al., 2014), rice, and other plant species (Powell et al., 1996; Temnykh et al., 2001). In many studies, the AG/CT di-nucleotide repeats was the most common for the transcribed regions (Kantety et al., 2002; Xu et al., 2012); similarly, GA/TC and AG/CT di-nucleotide repeats occurred at a higher frequency in the $C$. gigantea genome in our study (Table 2). We found that GA/TC and AG/CT account for $31.749 \%$ and $31.505 \%$ of all dinucleotide motifs, respectively. In our study, most tri-nucleotide repeats were TTC (Table 2), which is inconsistent with the reports for barley, maize, rice, sorghum (Kantety et al., 2002), and rubber (Feng et al., 2009). The most abundant tri-nucleotide motif was GGC/CCG in barley, maize, rice, and sorghum and AAC/TTG in wheat; the majority of tri-nucleotide repeats in rubber were AAG/CTT. 
Table 3. Characterization of six newly developed polymorphism genomic-derived simple sequence repeats (SSRs) in Colocasia gigantea.

\begin{tabular}{|c|c|c|c|c|c|}
\hline Primer & Sequence $\left(5^{\prime}-3^{\prime}\right)$ & Repeat motif & $\begin{array}{l}\text { PCR product } \\
\text { size (bp) }\end{array}$ & $\mathrm{Ta}\left({ }^{\circ} \mathrm{C}\right)$ & $\begin{array}{c}\text { GenBank } \\
\text { accession number }\end{array}$ \\
\hline \multirow[t]{2}{*}{ H4311UH01A8IX1 } & F: ATAGCTTCATGTGCATACCC & $(\mathrm{GA})^{16}$ & 127 & 55 & KR010361 \\
\hline & R: CCATCTCTCCACCGTTACTA & & & & \\
\hline \multirow[t]{2}{*}{ H4311UH01DFSZR } & F: TTGTTCATCCTACGGATTTC & $(\mathrm{TG})^{28}$ & 127 & 55 & KR010362 \\
\hline & R: ATGTTTCCATGCATTGTCTT & & & & \\
\hline \multirow[t]{2}{*}{ H4311UH01EM3GH } & F: TCGTAATTAACCGTAACGAAA & $(\mathrm{TC})^{18}$ & 178 & 55 & KR010363 \\
\hline & R: CAAGAGTGTTCGTCTTGGAC & & & & \\
\hline \multirow[t]{2}{*}{ H4311UH01DQIBR } & F: CAAGTTTGTGGGAAATTCAT & $(\mathrm{TTG})^{15}$ & 169 & 55 & KR010364 \\
\hline & R: ATCAAGTACAAAGAGGCAACA & & & & \\
\hline \multirow{2}{*}{ H4311UH01DCJBJ } & F: GAAGAAGAGCCACCAAGAAT & $(\mathrm{CA})^{19}$ & 172 & 55 & KR010365 \\
\hline & R: GTTTAAGAATGCGCCACTT & & & & \\
\hline \multirow[t]{2}{*}{ H4311UH01EW29I } & F: TТССТСТСТTТСТТССТССТT & $(\mathrm{CA})^{16}$ & 102 & 55 & KR010366 \\
\hline & R: GAGAGTGTGGGGGAAAAG & & & & \\
\hline
\end{tabular}

Ta: Annealing temperature.

Table 4. Genetic diversity parameters for 10 Colocasia gigantea.

\begin{tabular}{lcc}
\hline & Number of alleles & PIC value \\
\hline P8 & 4 & 0.615 \\
P29 & 3 & 0.732 \\
P37 & 6 & 0.756 \\
P41 & 7 & 0.621 \\
P59 & 3 & 0.561 \\
P73 & 5 & 0.689 \\
\hline
\end{tabular}

PIC: Polymorphic information content.

In the present study, the rest of the 24 amplifiable markers yielded at least two bands that could not be reliably scored; this could perhaps be the result of primer design or heterozygosis of $C$. gigantea in the tested population or an amplification area containing larger introns. This first set of microsatellite markers developed for C. gigantea will be useful for assessing genetic diversity and understanding the population structure of wild populations of $C$. gigantea and the Araceae family in general.

\section{CONCLUSION}

Colocasia gigantea is a very important vegetable in China that contains high dietary fiber, pyridoxine, and nicotinamide. Prior to this study, none of the public databases provided sequencing information for C. gigantea. We adopted the 454 sequencing technology to analyze the $C$. gigantea genome, characterized it by de novo sequencing without the presence of a reference genome, and developed a set of polymorphic simple sequence repeats (SSRs).

\section{ACKNOWLEDGEMENTS}

This work was supported by the National Science Foundation of China (31600254), National Science Foundation of Jiangsu Province of China (BK20150491), Start-up Fund for Advanced Talents of Jiangsu University (14JDG150), School of Agricultural Equipment Engineering at Jiangsu University, and the Priority Academic Program Development of Jiangsu Higher Education Institutions (PAPD, [2014]37).

\section{REFERENCES}

Angeloni, F., Wagemaker, C.A., Jetten, M.S., Op den Camp, H.J., Janssen-Megens, E.M., Francoijs, K.J., et al. 2011. De novo transcriptome characterization and development of genomic tools for Scabiosa columbaria L. using nextgeneration sequencing techniques. Molecular Ecology Resources 11:662-674. doi:10.1111/j.1755-0998.2011.02990.x. 
Bai,T.D.,Xu,L.A.,Xu,M., and Wang,Z.R. 2014. Characterization of masson pine (Pinus massoniana Lamb.) microsatellite DNA by 454 genome shotgun sequencing Tree. Genetics and Genomes 10:429-437. doi:10.1007/s11295-013-0684-y.

Baldwin, S., Pither-Joyce, M., Wright, K., Chen, L., and McCallum, J. 2012. Development of robust genomic simple sequence repeat markers for estimation of genetic diversity within and among bulb onion (Allium cepa L.) populations. Molecular Breeding 30(3):1401-1411. doi:10.1007/s11032-012-9727-6.

Botstein, D., White, R.L., Skolnick, M., and Davis, R.W. 1980. Construction of genetic linkage map in man using restriction fragment length polymorphisms. American Journal of Human Genetics 32(3):314-331.

Bown, D. 2000. Aroids: Plants of the Arum family. Vol. 2. Timber Press, London, UK.

Dellaporta, S.L., Wood, J., and Hicks, J.B. 1983. A plant DNA minipreparation: Version II. Plant Molecular Biology Reporter 1(4):19-21. doi:10.1007/BF02712670.

Egan, A.N., Schlueter, J., and Spooner, D.M. 2012. Applications of next-generation sequencing in plant biology. American Journal of Botany 99:175-185. doi:10.3732/ajb.1200020.

Feng, S., Li, W., Huang, H., Wang, J., and Wu, Y. 2009. Development, characterization and cross-species/genera transferability of EST-SSR markers for rubber tree (Hevea brasiliensis). Molecular Breeding 23:85-97. doi:10.1007/ s11032-008-9216-0.

Georgi, L., Herai, R.H., Vidal, R., Carazzolle, M.F., Pereira, G.G., Polashock, J., et al. 2012. Cranberry microsatellite marker development from assembled next-generation genomic sequence. Molecular Breeding 30:227-237. doi:10.1007/s11032-011-9613-7.

Ho, C.W., Wu, T.H., Hsu, T.W., Huang, J.C., Huang, C.C., and Chiang, T.Y. 2011. Development of 12 genic microsatellite loci for a biofuel grass, Miscanthus sinensis (Poaceae). American Journal of Botany 98:e201-e203. doi:10.3732/ ajb.1100071.

Ivancic, A., Roupsard, O., Garcia, J.Q., Melteras, M., Molisale, T., Tara, S., et al. 2008. Thermogenesis and flowering biology of Colocasia gigantea, Araceae. Journal of Plant Research 121:73-82. doi:10.1007/s10265-007-0129-5.

Kale, S.M., Pardeshi, V.C., Kadoo, N.Y., Ghorpade, P.B., Jana, M.M., and Gupta, V.S. 2012. Development of genomic simple sequence repeat markers for linseed using next-generation sequencing technology. Molecular Breeding 30:597606. doi:10.1007/s11032-011-9648-9.

Kantety, R.V., La Rota, M., Matthews, D.E., and Sorrells, M.E. 2002. Data mining for simple sequence repeats in expressed sequence tags from barley, maize, rice, sorghum and wheat. Plant Molecular Biology 48:501-510. doi:10.1023/A:1014875206165.

Li, D., Deng, Z., Qin, B., Liu, X., and Men, Z. 2012. De novo assembly and characterization of bark transcriptome using Illumina sequencing and development of EST-SSR markers in rubber tree (Hevea brasiliensis Muell. Arg.) BMC Genomics 13:192. doi:10.1186/1471-2164-13-192.

Nguyen, L.T. 2005. Bac há (Colocasia gigantea [Blume] Hook. F.) in the culinary history of Vietnamese-Americans. Economic Botany 59:185-190.

Perry, J.C., and Rowe, L. 2011. Rapid microsatellite development for water striders by next-generation sequencing. Journal of Heredity 102:125-129. doi:10.1093/jhered/esq099.

Powell, W., Machray, G.C., and Provan, J. 1996. Polymorphism revealed by simple sequence repeats. Trends in Plant Science 1:215-222. doi:10.1016/1360-1385(96)86898-1.

Raymond, M., and Rousset, F. 1995. GENEPOP (version 1.2): Population genetics software for exact tests and ecumenicism. Journal of Heredity 86:248-249.

Sahu, B., Patel, A., Sahoo, L., Das, P., Meher, P., and Jayasankar, P. 2012. Rapid and cost effective development of SSR markers using next generation sequencing in Indian major carp, Labeo rohita (Hamilton, 1822). Indian Journal of Fisheries 59(3):21-24.

Temnykh, S., DeClerck, G., Lukashova, A., Lipovich, L., Cartinhour, S., and McCouch, S. 2001. Computational and experimental analysis of microsatellites in rice (Oryza sativa L.): Frequency, length variation, transposon associations, and genetic marker potential. Genome Research 11:1441-1452. doi:10.1101/gr.184001.

Wang, Z., Fang, B., Chen, J., Zhang, X., Luo, Z., Huang, L., et al. 2010. De novo assembly and characterization of root transcriptome using Illumina paired-end sequencing and development of cSSR markers in sweetpotato (Ipomoea batatas). BMC Genomics 11:726. doi:10.1186/1471-2164-11-726.

Xu, W., Yang, Q., Huai, H., and Liu, A. 2012. Development of EST-SSR markers and investigation of genetic relatedness in tung tree. Genetics and Genomes 8:933-940. doi:10.1007/s11295-012-0481-z.

Yang, H., Tao, Y., Zheng, Z., Li, C., Sweetingham, M.W., and Howieson, J.G. 2012. Application of next-generation sequencing for rapid marker development in molecular plant breeding: A case study on anthracnose disease resistance in Lupinus angustifolius L. BMC Genomics 13:318. doi:10.1186/1471-2164-13-318.

Zalapa, J.E., Cuevas, H., Zhu, H., Steffan, S., Senalik, D., Zeldin, E., et al. 2012. Using next-generation sequencing approaches to isolate simple sequence repeat (SSR) loci in the plant sciences. American Journal of Botany 99:193-208. doi:10.3732/ajb.1100394. 